Gut and Liver, Vol. 11, No. 3, May 2017, pp. 434-439

\title{
The Role of Endoscopic Biliary Drainage without Sphincterotomy in Gallstone Patients with Cholangitis and Suspected Common Bile Duct Stones Not Detected by Cholangiogram or Intraductal Ultrasonography
}

\author{
Hyeon Jeong Goong ${ }^{1}$, Jong Ho Moon ${ }^{1}$, Yun Nah Lee ${ }^{1}$, Hyun Jong Choi ${ }^{1}$, Seo-Youn $\mathrm{Choi}^{2}$, Moon Han Choi ${ }^{1}$, Min Jin Kim , \\ Tae Hoon Lee ${ }^{1}$, Sang-Heum Park ${ }^{1}$, and Hae Kyung Lee ${ }^{2}$ \\ ${ }^{\prime}$ Digestive Disease Center and Research Institute, Department of Internal Medicine, SoonChunHyang University School of Medicine, Bucheon \\ and Seoul, and ${ }^{2}$ Department of Radiology, SoonChunHyang University School of Medicine, Bucheon, Korea
}

Background/Aims: Treatment for cholangitis without common bile duct (CBD) stones has not been established in patients with gallstones. We investigated the usefulness of endoscopic biliary drainage (EBD) without endoscopic sphincterotomy (EST) in patients diagnosed with gallstones and cholangitis without CBD stones by endoscopic retrograde cholangiopancreatography (ERCP) and intraductal ultrasonography (IDUS). Methods: EBD using $5 \mathrm{~F}$ plastic stents without EST was performed prospectively in patients with gallstones and cholangitis if CBD stones were not diagnosed by ERCP and IDUS. After ERCP, all patients underwent laparoscopic cholecystectomy. The primary outcomes were clinical and technical success. The secondary outcomes were recurrence rate of biliary events and procedure-related adverse events. Results: Among 187 patients with gallstones and cholangitis, 27 patients without CBD stones according to ERCP and IDUS received EBD using $5 \mathrm{~F}$ plastic stents without EST. The stents were maintained in all patients until laparoscopic cholecystectomy, and recurrence of cholangitis was not observed. After cholecystectomy, the stents were removed spontaneously in 12 patients and endoscopically in 15 patients. Recurrence of CBD stones was not detected during the follow-up period (median, 421 days). Conclusions: EBD using $5 \mathrm{~F}$ plastic stents without EST may be safe and effective for the management of cholangitis accompanied by gallstones in patients without CBD stones according to ERCP and IDUS. (Gut Liver 2017;11:434-439)

Key Words: Cholangitis; Gallstones; Sphincterotomy, endoscopic; Intraductal ultrasonography

\section{INTRODUCTION}

The prevalence of common bile duct (CBD) stones accompanying gallstones has been reported in 10\% to 15\% of cases, and the presence of cholangitis in patients with symptomatic cholelithiasis is one of the leading predictors of choledocholithiasis. ${ }^{1}$ Therefore, CBD is usually evaluated before cholecystectomy, because remnant CBD stones can cause biliary and pancreatic problems. When CBD stones are confirmed by imaging studies, endoscopic management can be performed. However, the procedure for further management of cases in whom the presence of CBD stones is not definite even after endoscopic retrograde cholangiopancreatography (ERCP), despite the high possibility on initial clinical findings, is unclear. In previous studies, ERCP detected $27 \%$ to $80 \%$ of CBD stones when the presence of a stone was highly suspected. ${ }^{2-4}$ Therefore, some reports suggested that endoscopic sphincterotomy (EST) can be performed in patients with negative findings on ERCP to prevent adverse effects resulting from undetected stones. However, the results of comparative studies are controversial. ${ }^{5,6}$ Furthermore, EST causes early and late complications, such as pancreatitis, perforation, bleeding, permanent loss of sphincter Oddi function, bacterial translocation in the bile duct, and recurrence of biliary colic. ${ }^{7-11}$

To prevent unnecessary EST, the presence of CBD stones should be confirmed. Intraductal ultrasonography (IDUS) provides high-quality, real-time, accurate images of the entire CBD without EST. ${ }^{12,13}$ In this study, we evaluated the efficacy of endoscopic biliary drainage using $5 \mathrm{~F}$ plastic stent without EST as a treatment for patients with cholangitis and gallstones showing negative result for CBD stones on ERCP and IDUS.

Correspondence to: Jong Ho Moon

Digestive Disease Center and Research Institute, Department of Internal Medicine, SoonChunHyang University Bucheon Hospital, SoonChunHyang University School of Medicine, 170 Jomaru-ro, Wonmi-gu, Bucheon 14584, Korea

Tel: +82-32-621-5094, Fax: +82-32-621-5080, E-mail: jhmoon@schmc.ac.kr

Received on May 7, 2016. Revised on July 4, 2016. Accepted on August 5, 2016. Published online January 20, 2017

pISSN 1976-2283 eISSN 2005-1212 https://doi.org/10.5009/gnl16234

@ This is an Open Access article distributed under the terms of the Creative Commons Attribution Non-Commercial License (http://creativecommons.org/licenses/by-nc/4.0) which permits unrestricted non-commercial use, distribution, and reproduction in any medium, provided the original work is properly cited. 


\section{MATERIALS AND METHODS}

\section{Patients}

Between March 2009 and June 2013, patients who were diagnosed with gallstone and cholangitis without CBD stones on ERCP were enrolled prospectively and analyzed retrospectively. The diagnosis of gallstones was confirmed by at least a single investigational modality: primarily ultrasonography (US), computed tomography (CT), magnetic resonance imaging (MRI) or EUS. Diagnosis of cholangitis was based on laboratory and clinical findings of abdominal pain, fever, and jaundice. The exclusion criteria were (1) presence of CBD stone revealed by investigational modalities, (2) previous EST or endoscopic pancreaticobiliary drainage, (3) previous biliopancreatic surgery including cholecystectomy, (4) periampullary malignancy, (5) recurrent CBD stones, (6) contraindication to ERCP. Our Institutional Review Board (IRB) approved this study (IRB approval number: SCHBC 2009-01-139-001), and written informed consent was obtained from all enrolled patients.

\section{Procedures}

\section{1) ERCP and IDUS for detection of CBD stones}

All patients were under conscious sedation during the procedure. Two experienced endoscopists performed ERCP using a conventional duodenoscope. The bile duct was evaluated using 50\% diluted contrast media (Omnipaque; GE Healthcare, Seoul, Korea) to identify floating stones. An endoscopist and a radiologist confirmed the absence of filling defect on fluoroscopy by examining the digital image. If there was no filling defect on ERCP, IDUS was performed to identify CBD stones. We examined the bile duct from the first branch of the intrahepatic duct to the papilla via wire-guided IDUS using a 2.0-mm diameter catheter US probe with a frequency of $20 \mathrm{MHz}$ (UM-G20-29R; Olympus Medical System, Co., Ltd., Tokyo, Japan). Two investigators evaluated the IDUS findings simultaneously. The positive finding of stones on IDUS was defined as any sized echogenic materials with posterior acoustic shadowing.

\section{2) Treatment for patients with or without CBD stones}

If stones were demonstrated by IDUS, EST was performed, and stone extraction was completed using a basket or balloon retrieval catheter. In patients who were not diagnosed with CBD stones on IDUS, a 5F (1.67 mm) plastic stent (length 9, 10, 12 $\mathrm{cm}$; double pigtail or straight type; Cook Medical, WinstonSalem, NC, USA) was inserted through the papilla to the first branch of intrahepatic bile duct without performing EST (Fig. 1). All patients were referred to surgery for laparoscopic cholecystectomy. The duration between ERCP and laparoscopic cholecystectomy was determined based on the patient's condition.

\section{Follow-up}

All of the patients routinely underwent follow-up investigations with laboratory testing and simple abdominal radiography after ERCP. Bleeding was defined as a decrease in hemoglobin level of $2 \mathrm{~g} / \mathrm{dL}$ or more compared with the baseline level, together with clinical evidence of bleeding. For patients with abdominal pain, serum amylase and lipase levels were measured, and an abdominal CT scan was performed if symptoms persisted. Acute pancreatitis was defined as abdominal pain coupled with a 3-fold elevation in serum amylase or lipase levels.

The stents were removed 4 to 8 weeks after cholecystectomy during endoscopic examination without performing ERCP. After removal of the stent, all of the patients were scheduled to visit our outpatient clinic every month for 6 months and then once again 6 months later. We investigated the recurrence rate of biliary colic, cholangitis and CBD stones. The follow-up period was defined from the date of ERCP to the date of the last visit to our outpatient clinic. Diagnosis of recurrent cholangitis was based on laboratory and clinical findings.
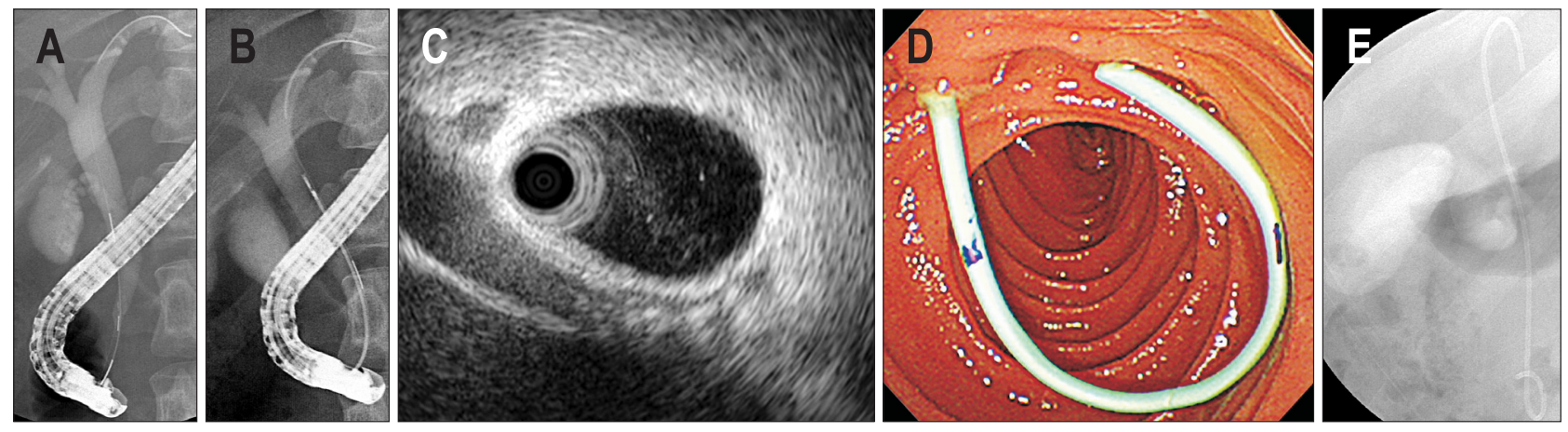

Fig. 1. Endoscopic biliary drainage using a 5 F plastic stent without endoscopic sphincterotomy (EST). Cholangiogram showing (A) no definite stone in a gallstone patient with cholangitis. (B) Intraductal ultrasonography (IDUS) was performed for the detection of common bile duct (CBD) stones. (C) IDUS showing negative finding of CBD stones or sludge. (D) Endoscopic view showing an inserted 5F plastic stent without EST. (E) Cholangiogram showing a $5 \mathrm{~F}$ plastic stent inserted into the bile duct. 


\section{Outcomes}

The primary outcomes of this study were clinical and technical success. Clinical success was defined as $>50 \%$ reduction in the total bilirubin level within 1 week without cholangitis. The secondary outcomes were recurrence rate of biliary events during the follow-up period, procedure-related adverse events, duration between ERCP and cholecystectomy, and hospital stay duration. The procedure-related adverse events were divided into pancreatitis, bleeding, perforation, and cholangitis.

\section{Statistical analysis}

Categorical parameters including sex, location of a mass, and technical details were expressed as frequencies and proportions. Age and initial laboratory findings were summarized as the mean \pm standard deviation. Durations from ERCP to cholecystectomy and from cholecystectomy to endoscopic stent removal were expressed as median (interquartile range, IQR). SPSS version 18.0 (SPSS Inc., Chicago, IL, USA) was used for the statistical analyses.

\section{RESULTS}

There were 187 patients with gallbladder stones and cholangitis, but no evidence of CBD stones on ERCP, who underwent IDUS to dectect CBD stones. Of these patients, 160 were excluded because of CBD stones on IDUS. In the final analysis, 27 patients (12 males, $46.9 \pm 15.1$ years) with cholangitis and gallstone were evaluated in this study after confirming no evidence of CBD stones using ERCP and IDUS (Fig. 2). The baseline characteristics of the 27 patients and initial laboratory findings are shown in Table 1. Although the median levels of amylase and lipase were within normal range, combined pancreatitis was suspected in eight symptomatic patients with more than 3-fold the upper normal limit of amylase and lipase levels.

\section{Outcomes of $5 \mathrm{~F}$ plastic stenting without sphincterotomy and follow-up before cholecystectomy}

The 5F plastic stenting without EST was performed successfully in all patients with a 100\% clinical success rate. Total bilirubin levels were reduced to within normal range in all but one patient. The median duration between ERCP and laparoscopic cholecystectomy was 7 days (IQR, 5 to 8 days), and the 5F stent was maintained in all patients until laparoscopic cholecystectomy. There was no recurrence of cholangitis until cholecystectomy after $5 \mathrm{~F}$ plastic stenting without sphincterotomy. No procedure-related adverse events were observed (Table 2).

\section{Outcomes of 5F stent removal and follow-up after cholecystectomy}

The median follow-up duration was 421 days (IQR, 137 to

Table 1. Baseline Characteristics and Initial Laboratory Findings $(\mathrm{n}=27)$

\begin{tabular}{lc}
\hline Characteristic & Value \\
\hline Male sex & $12(44.4)$ \\
Age, yr & $46.9 \pm 15.1$ \\
Clinical symptoms & \\
Abdominal pain & $27(100)$ \\
Jaundice & $21(77.7)$ \\
Fever & $22(81.4)$ \\
Initial laboratory findings & \\
Total bilirubin, mg/dL & $3.79 \pm 3.08$ \\
AST, IU/L & $411.62 \pm 400.04$ \\
ALT, IU/L & $385.88 \pm 352.51$ \\
GGT, IU/L & $582.81 \pm 522.55$ \\
\hline
\end{tabular}

Quantitative data are presented as number $(\%)$ or mean \pm SD.

AST, aspartate aminotransferase; IU, international unit; ALT, alanine aminotransferase; GGT, $\gamma$-glutamyl transpeptidase.

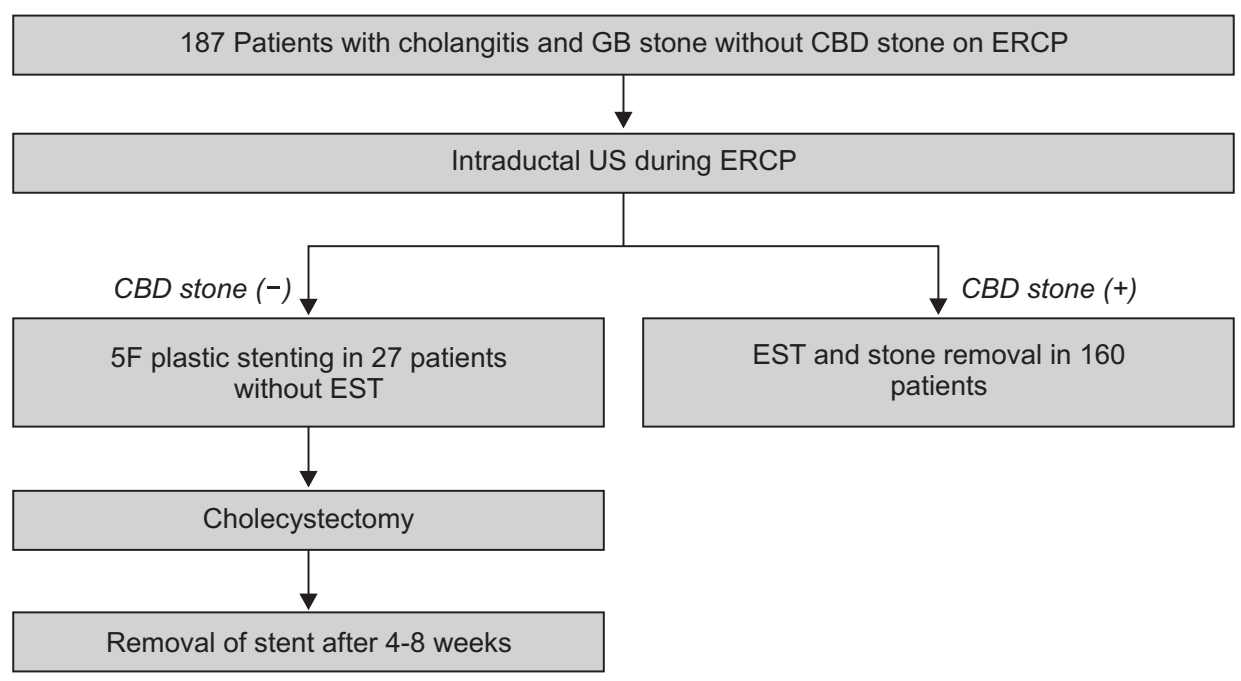

Fig. 2. Flowchart of enrolled patients.

$\mathrm{GB}$, gallbladder; $\mathrm{CBD}$, common bile duct; ERCP, endoscopic retrograde cholangiopancreatography; US, ultrasonography; EST, endoscopic sphincterotomy. 
Table 2. Outcomes of 5F Plastic Stenting without Endoscopic Sphincterotomy and Follow-up before Cholecystectomy $(\mathrm{n}=27)$

\begin{tabular}{lc}
\hline \multicolumn{1}{c}{ Characteristic } & Value \\
\hline Technical success & $27(100)$ \\
Clinical success & $27(100)$ \\
Adverse events & 0 \\
Follow-up between 5F plastic stenting and cholecystectomy \\
$\quad$ Duration until cholecystectomy, days & $7(5-8)$ \\
Dislodgement of stent before cholecystectomy & 0 \\
New onset and aggravation of biliary problems & 0 \\
Normalization of bilirubin after 5F plastic stenting & $26(96.3)$ \\
\hline
\end{tabular}

Qualitative data and quantitative data are presented as number $(\%)$ and median (interquartile range), respectively.

517 days). The $5 \mathrm{~F}$ stent was removed successfully during the follow-up period after cholecystectomy in all patients. The spontaneous stent removal occurred in 12 patients (44.4\%) after cholecystectomy. The other 15 patients (55.6\%) underwent endoscopic removal of the stent after a median of 50 days (IQR, 58 to 109 days) from the operation. During the follow-up period, recurrent cholangitis developed in one patient (3.7\%) whose stent removal was delayed for 6 months. Other additional biliary events did not occur (Table 3).

\section{DISCUSSION}

Previous reports have demonstrated that 10\% to $15 \%$ of gallstones occur with the presence of CBD stones. There are several predictors of choledocholithiasis with symptomatic cholelithiasis such as cholangitis, laboratory findings (bilirubin $>4 \mathrm{mg} / \mathrm{dL}$ ), and radiological findings. The presence of cholangitis is a very strong predictor of accompanying CBD stones ${ }^{14}$ that affect the postoperative prognosis. Therefore, preoperative ERCP is recommended in patients with highly suspected CBD stones, especially cholangitis. When ERCP shows CBD stones, endoscopic removal with EST is performed. However, there are no current treatment guidelines established for cases in which stones are not seen by ERCP, despite the suspicion of CBD stones in pre-ERCP findings.

Brand et al. ${ }^{2}$ suggested that EST can be performed in patients presenting typical clinical symptoms associated with biliary origin despite inconclusive ERCP findings. The authors insisted that ERCP is not sensitive for detection of microlithiasis. According to previous data, ERCP detects CBD stones in 27\% to $80 \%$ of cases, even in highly suspicious choledocholithiasis based on clinical, laboratory, and radiological findings., ${ }^{3,4}$ The existence of undetected small stones can cause biliary problems as severe as those in cases with large stones. Therefore, EST during ERCP has often been considered to prevent complications resulting from unseen stones on ERCP. On the other hand, the results of previous comparative studies are controversial. Siddique et al. ${ }^{5}$ reported that EST is able to increase the detection rate of small
Table 3. Outcomes of 5F Stent Removal and Follow-up after Cholecystectomy $(\mathrm{n}=27)$

\begin{tabular}{ll}
\hline \multicolumn{1}{c}{ Characteristic } & \multicolumn{1}{c}{ Value } \\
\hline Follow-up duration after cholecystectomy, day & $421(137-517)$ \\
Recurrent biliary events & $1(3.7)$ \\
Recurrent cholangitis & 1 \\
Recurrent CBD stones & 0 \\
Recurrent biliary colic & 0 \\
Stent removal & $27(100)$ \\
Spontaneous dislodgement & $12(44.4)$ \\
Endoscopic removal & $15(55.6)$ \\
Time to endoscopic stent removal & $50(58-109)$ \\
after cholecystectomy, days & \\
\hline
\end{tabular}

Quantitative data are presented as median (interquartile range). CBD, common bile duct.

CBD stones or debris in patients with normal findings on ERCP before cholecystectomy for symptomatic gallstones. However, this trial showed no differences in clinical outcomes after cholecystectomy between the EST and non-EST groups despite the increased detection rate of undetected stones. ${ }^{5}$ Also, Hui et al. ${ }^{6}$ randomized 111 patients suffering from acute cholangitis and gallbladder stones without evidence of CBD stones on ERCP into EST and non-EST groups. In this study, EST decreased the duration of acute cholangitis and the length of hospital stay but did not decrease the incidence of recurrent cholangitis.

EST is an independent risk factor for complications during ERCP. ${ }^{7,8}$ Also, the long-term outcome of EST has been of concern. ${ }^{9,10}$ Fujimoto et al. ${ }^{11}$ followed up 522 patients who received EST due to choledocholithiasis and cholelithiasis for 5.6 years. Sphincter dysfunction causes duodenobiliary reflux and intestinal content reflux that result in recurrent choledocholithiasis, cholecystitis, inflammation in the bile duct, and biliary malignancy. Therefore, it is recommended that the use of EST should be limited to cases with adequate indication based on accurate diagnostic tools. IDUS has shown a superior rate of detection of small stones compared with other imaging modalities. It can be performed easily during ERCP and provide real-time, crosssectional images of the entire bile duct. ${ }^{12,13}$ In our study, we evaluated whether CBD stones are seen in the bile duct using IDUS at least twice in patients with symptomatic gallbladder stones having cholangitis, which is a very strong factor for accompanying CBD stones. Therefore, we could avoid unnecessary EST by confirming the absence of biliary materials using IDUS, followed by transient insertion of a $5 \mathrm{~F}$ plastic stent to maintain biliary drainage, including sludge and remnant contrast, after the procedure. Persistent inflammation in the bile duct due to purulent bile and remnant media after ERCP results in postoperative complications such as leakage. ${ }^{15}$ Conservative treatment alone with antibiotics usually resolves inflammation in 70\% of patients, but the remaining 30\% with persisting inflammation 
might experience complications.

Transient endoscopic biliary drainage using a plastic stent is one of the methods used to relieve cholestasis in cholangitis, and it is easily performed compared with EST and the plastic stent can be safely removed. ${ }^{16}$ Therefore, we performed the insertion of plastic stents before cholecystectomy. As a result, the clinical and laboratory findings improved completely before surgery in all patients except one. That patient was a 30-yearold female with an initial total bilirubin level of $16.42 \mathrm{mg} / \mathrm{dL}$ and a preoperative level of $2.24 \mathrm{mg} / \mathrm{dL}$. She had combined acute pancreatitis and sepsis. No patient experienced new symptoms or aggravation of biliary problems during the admission period.

A plastic stent in the bile duct may cause additional problems, such as obstruction, infection, or fibrotic changes in the bile duct wall. However, Verzhbitsky et al. ${ }^{15}$ compared the results of laparoscopic cholecystectomy between an EST with biliary stent group and an EST without stent group in 162 patients. In their study, group prophylactic plastic stent insertion had no impact on biliary complications. In our study, there were no problems due to plastic catheter placement during the follow-up period. We planned to remove the inserted stents 2 months after cholecystectomy to avoid long-term side effects. All stents were spontaneously or intentionally removed during the scheduled follow-up, with the exception of one patient who missed the appointment. This stent remained in the bile duct for 180 days after cholecystectomy, resulting in a total bilirubin level of up to $3 \mathrm{mg} / \mathrm{dL}$ that did not cause any symptoms. A CBD stone was not detected on ERCP with IDUS, and the stent was removed, resulting in laboratory findings within normal limits. The patient was suspected of having recurrent cholangitis due to the long duration of plastic stent placement without recurrent CBD stone, because there was no biliary colic or radiologic evidence of CBD stone.

In determining whether endoscopists should perform EST, the most important concern is recurrent biliary events such as recurrent biliary stone, recurrent cholangitis, and biliary colic during long-term follow-up. Lee et al. ${ }^{17}$ suggested that EST reduces further episodes of cholangitis in patients with current cholangitis and the high probability of choledocholithiasis despite inconclusive ERCP findings. In that study, the cumulative rate of recurrent cholangitis was less in the EST group than non-EST group. Recurrent cholangitis occurred in 6.0\% of patients (5/83) in the EST group, of which four cases were due to choledocholithiasis. Although this study did not determine independent factors for recurrent cholangitis, cholecystectomy was not performed in every patient with gallbladder stones, and the bile duct was evaluated by ERC alone. Biliary complications were reported in $3.7 \%$ to $24 \%$ of patients even after complete removal of CBD stones via EST. ${ }^{14,18,19}$ The independent factors proven to cause recurrent biliary problems after endoscopic procedures include dilated bile duct, gallbladder in situ, gallbladder stone in situ, periampullary diverticulum, papillary stenosis, ${ }^{19-21}$ as well as overlooked CBD stones. Two studies showed that detecting small stones using IDUS can reduce the recurrence of biliary disease. $^{22,23}$ We evaluated the bile duct using ERCP and IDUS. Furthermore, our patients received laparoscopic cholecystectomy for symptomatic gallstones. As a result, recurrent biliary events occurred in only one patient, who had a remnant stent for 180 days. Therefore, when the possibility of remaining undetected stones is reduced by IDUS in patients expecting gallbladder removal, EST for prevention of additional biliary problems can be avoided by transient plastic stenting.

There are several limitations in this study. First, although the findings of no evidence for stones on ERCP and IDUS were evaluated by clinical follow-up for more than 6 months, a true negative gold standard would have consisted of EST followed by extraction trials using a balloon or basket catheter. However, we did not performed EST only for the confirmation of our previous findings in patients with no evidence of stones by IDUS. Additionally, IDUS cannot be used to interrogate intrahepatic bile ducts (IHDs) fully because of the technical difficulty of probe insertion into each of the narrow branches. In the present study, we inserted the probe only up to the first branch of the IHD. Therefore, even if recurrent cholangitis within 6 months after ERCP is not observed, it would be difficult to conclude that there were no stones in IHDs. A 5F plastic stent for ERBD was used without EST, but there was no evidence that a $5 \mathrm{~F}$ plastic stent was more effective than the other sized plastic stents. However, a $5 \mathrm{~F}$ plastic stent may reduce the risk of developing post-procedural pancreatitis and the potential of spontaneous removal compared with a stent of larger diameter. Another limitation was the small sample size, which limited the statistical strength of the study. To confirm our results, further studies making comparisons with conventional treatment using EST should be performed in a larger cohort and for a longer period.

In conclusion, IDUS and endoscopic biliary drainage using $5 \mathrm{~F}$ plastic stents can be used safely and effectively for cholangitis accompanying gallstones without definite CBD stone on ERCP to avoid unnecessary EST before cholecystectomy.

\section{CONFLICTS OF INTEREST}

No potential conflict of interest relevant to this article was reported.

\section{ACKNOWLEDGEMENTS}

We thank A Ri Song, RN, Song Ah Jeong, RN, and Sun Yeong Moon, RN, and the rest of the nursing staff for assistance with the procedures. This work was supported, in part, by the SoonChunHyang University Research Fund. 


\section{REFERENCES}

1. ASGE Standards of Practice Committee, Maple JT, Ben-Menachem $\mathrm{T}$, et al. The role of endoscopy in the evaluation of suspected choledocholithiasis. Gastrointest Endosc 2010;71:1-9.

2. Brand B, Wiese L, Thonke F, et al. Outcome of endoscopic sphincterotomy in patients with pain of suspected biliary or papillary origin and inconclusive cholangiography findings. Endoscopy 2001;33:405-408.

3. Lee YT, Chan FK, Leung WK, et al. Comparison of EUS and ERCP in the investigation with suspected biliary obstruction caused by choledocholithiasis: a randomized study. Gastrointest Endosc 2008;67:660-668.

4. Chak A, Hawes RH, Cooper GS, et al. Prospective assessment of the utility of EUS in the evaluation of gallstone pancreatitis. Gastrointest Endosc 1999;49:599-604.

5. Siddique I, Mohan K, Khajah A, et al. Sphincterotomy in patients with gallstones, elevated LFTs and a normal CBD on ERCP. Hepatogastroenterology 2003;50:1242-1245.

6. Hui CK, Lai KC, Wong WM, Yuen MF, Lam SK, Lai CL. A randomised controlled trial of endoscopic sphincterotomy in acute cholangitis without common bile duct stones. Gut 2002;51:245247.

7. ASGE Standards of Practice Committee, Anderson MA, Fisher L, et al. Complications of ERCP. Gastrointest Endosc 2012;75:467-473.

8. Loperfido S, Angelini G, Benedetti G, et al. Major early complications from diagnostic and therapeutic ERCP: a prospective multicenter study. Gastrointest Endosc 1998;48:1-10.

9. Prat F, Malak NA, Pelletier G, et al. Biliary symptoms and complications more than 8 years after endoscopic sphincterotomy for choledocholithiasis. Gastroenterology 1996;110:894-899.

10. Sugiyama M, Atomi Y. Risk factors predictive of late complications after endoscopic sphincterotomy for bile duct stones: longterm (more than 10 years) follow-up study. Am J Gastroenterol 2002;97:2763-2767.

11. Fujimoto T, Tsuyuguchi T, Sakai Y, et al. Long-term outcome of endoscopic papillotomy for choledocholithiasis with cholecystolithiasis. Dig Endosc 2010;22:95-100.

12. Kim HS, Moon JH, Choi HJ, et al. The role of intraductal US in the management of idiopathic recurrent pancreatitis without a definite cause on ERCP. Gastrointest Endosc 2011;73:1148-1154.

13. Yoon LY, Moon JH, Choi HJ, et al. Clinical usefulness of intraductal ultrasonography for the management of acute biliary pancreatitis. J Gastroenterol Hepatol 2015;30:952-956.

14. Bergman JJ, van der Mey S, Rauws EA, et al. Long-term followup after endoscopic sphincterotomy for bile duct stones in patients younger than 60 years of age. Gastrointest Endosc 1996;44:643649.

15. Verzhbitsky V, Zeina AR, Depsames R, Ovadia B, Atia O, Fireman Z. Does prophylactic stent insertion to the common bile duct during endoscopic retrograde cholangiopancreatography (ERCP) before cholecystectomy have any impact on the rate of biliary complications? Surg Endosc 2013;27:4620-4624.

16. Nagino M, Takada T, Kawarada Y, et al. Methods and timing of biliary drainage for acute cholangitis: Tokyo Guidelines. J Hepatobiliary Pancreat Surg 2007;14:68-77.

17. Lee SH, Hwang JH, Yang KY, et al. Does endoscopic sphincterotomy reduce the recurrence rate of cholangitis in patients with cholangitis and suspected of a common bile duct stone not detected by ERCP? Gastrointest Endosc 2008;67:51-57.

18. Hawes RH, Cotton PB, Vallon AG. Follow-up 6 to 11 years after duodenoscopic sphincterotomy for stones in patients with prior cholecystectomy. Gastroenterology 1990;98:1008-1012.

19. Kim DI, Kim MH, Lee SK, et al. Risk factors for recurrence of primary bile duct stones after endoscopic biliary sphincterotomy. Gastrointest Endosc 2001;54:42-48.

20. Geenen JE, Toouli J, Hogan WJ, et al. Endoscopic sphincterotomy: follow-up evaluation of effects on the sphincter of Oddi. Gastroenterology 1984;87:754-758.

21. Tanaka M, Takahata S, Konomi H, et al. Long-term consequence of endoscopic sphincterotomy for bile duct stones. Gastrointest Endosc 1998;48:465-469.

22. Tsuchiya S, Tsuyuguchi T, Sakai Y, et al. Clinical utility of intraductal US to decrease early recurrence rate of common bile duct stones after endoscopic papillotomy. J Gastroenterol Hepatol 2008;23:1590-1595.

23. Ohashi A, Tamada K, Wada S, et al. Risk factors for recurrent bile duct stones after endoscopic papillary balloon dilation: long-term follow-up study. Dig Endosc 2009;21:73-77. 